\title{
Un moment pour réfléchir
}

\author{
Douglas McDougall ${ }^{1}$
}

Accepted: 20 December 2021/Published online: 8 January 2022

(C) Ontario Institute for Studies in Education (OISE) 2022

L'année nous offre de maintes occasions de nous arrêter pour réfléchir à ce qui vient de se passer sur une certaine période. La fin d'une année civile représente pour une grande majorité d'entre nous le moment propice pour nous rappeler ce qui a été accompli dans l'année et même pour penser à ce que nous pourrions réaliser dans l'année qui vient. Ce numéro est plutôt unique en son genre. Tous les articles sauf un seul sont le fruit de chercheurs issus d'universités canadiennes. Vous êtes invités à soumettre une réponse à l'un des articles dans le but de poursuivre la discussion sur les thèmes et enjeux qui concernent l'enseignement des sciences, des mathématiques et de la technologie.

Chourney et Bakos (2021) examinent les récits d'enseignants non autochtones en formation initiale pour savoir comment ils conçoivent l'appel visant à inclure la vision du monde et les perspectives des Premiers Peuples dans leur enseignement des mathématiques. Ces enseignants en formation initiale sont inscrits à un cours de premier cycle de préparation du personnel enseignant qui explore les façons d'harmoniser l'enseignement des mathématiques avec le savoir, les pédagogies et les points de vue autochtones. Les auteurs ont examiné les réflexions notées par les étudiants au début et à la fin du cours en utilisant la théorie du positionnement dans le but de déterminer les tendances et les thèmes ressortis. Chorney a identifié des exemples de recadrage, de retemporisation et de repersonnalisation mathématiques. Ces enseignants en formation initiale se sont montrés disposés à repenser leurs positions personnelles. L'autochtonisation de la formation des enseignants en mathématiques comporte de nombreux défis. Cette étude peut servir à aider les enseignants en formation initiale et le personnel enseignant universitaire à entamer un dialogue productif sur les moyens d'appuyer les futurs enseignants non autochtones.

Husband (2021) se penche sur des enseignants en formation initiale alors qu'ils suivent un cours de perfectionnement professionnel d'une durée de 10 jours après avoir complété leur programme de formation à l'enseignement. On a demandé aux enseignants de réaliser des tâches mathématiques du niveau scolaire primaire. On a analysé les données vidéo ainsi que les carnets des futurs enseignants en utilisant la théorie pour la croissance dynamique de la compréhension mathématique

Douglas McDougall

doug.mcdougall@utoronto.ca

1 Ontario Institute for Studies in Education, University of Toronto, Ontario M5S 1V6 Toronto, Canada 
Pirie et Kieren (1994). Dans cet article, Husband se concentre sur la façon dont les enseignants en formation initiale conçoivent la soustraction. Il constate qu'il est difficile pour les enseignants de décortiquer et de bien utiliser les connaissances existantes des mathématiques. Le processus a exigé une longue observation et des interventions ponctuelles utiles qui ont exigé beaucoup de temps et de stratégies d'enseignement. Cette étude montre que les futurs enseignants ont besoin de plus de cours de perfectionnement professionnel et d'activités leur permettant d'explorer et d'accroître leur compréhension des mathématiques du primaire.

Throope Robinson et al. (2021) explorent l'incidence d'un programme de perfectionnement professionnel sous la forme d'un certificat d'une durée de deux ans en pédagogie des mathématiques du primaire sur la capacité des éducateurs à enseigner les mathématiques dans les écoles primaires. Leur étude à méthodes de recherche mixtes a été effectuée à l'aide d'un sondage en ligne et des entrevues avec les enseignants et elle visait à déterminer l'efficacité du programme et à mesurer le niveau de préparation des enseignants à assumer un rôle de chef de file en enseignement des mathématiques. Trois thèmes sont ressortis: le perfectionnement professionnel et la connaissance de la matière, le leadership et une pédagogie qui tient compte de la culture. L'un des objectifs consistait à renforcer la capacité des enseignants et il a été noté que pour nourrir le développement de relations constructives et établir un réseau, il est nécessaire d'avoir des entretiens réguliers avec les enseignants. Les auteurs reconnaissent l'importance d'offrir aux enseignants une formation ciblée leur permettant de différencier l'éducation et l'évaluation et acquérir une meilleure compréhension des talents et expériences que les élèves apportent en classe. Il existe plusieurs moyens de renforcer les compétences en leadership des enseignants dans les écoles primaires et ce programme de certificat s'est avéré un succès pour former des chefs de file dans l'enseignement des mathématiques.

Saldanha et Hatfield (2021) se sont intéressés à l'utilisation du logiciel TinkerPlots ${ }^{\mathrm{MC}}$ par des élèves de $7^{\mathrm{e}}$ année dans le but d'organiser des ensembles de données. La séquence pédagogique était conçue de façon à ce qu' elle les aide à comprendre l'emplacement des données organisées en quartiles à l'intérieur d'un intervalle de variation et la répartition de ces portions. On a administré un prétest aux élèves puis assigné une tâche intégratrice dans le but de faire ressortir des données hypothétiques résultant d'un diagramme de quartiles. On a constaté que les élèves confondaient la signification de la localisation d'un quartile avec sa répartition. Ceci montre que l'attention des élèves n'était pas divisée également entre la localisation et la répartition des parties de données. Cette étude nous aide à mieux comprendre les tâches éducatives qui peuvent servir à orienter l'attention des élèves dans la localisation et la répartition des données.

Anwandter Cuellar et al. (2021) présentent les résultats de la recherche portant sur la pensée algébrique des enfants de trois à huit ans. Les résultats montrent que des problèmes d'ordre conceptuel nuisent à la caractérisation de la pensée algébrique. Cependant, on constate que les jeunes enfants possèdent un grand potentiel pour comprendre des aspects relevant de la pensée algébrique. Cet article montre qu'il y a encore de nombreuses questions sans réponse en ce qui a trait au mode de développement du stade précoce de la pensée algébrique et le moment où il se manifeste chez les enfants de trois à huit ans.

À l'intérieur du thème central du numéro 21.2, on retrouvait des articles qui portaient sur les façons de «répondre au racisme anti-noir dans l'enseignement des sciences, des mathématiques, de la technologie et des STIM». Dans la foulée de ce thème, Davis (2021) se penche sur la démarche d'enseignement des mathématiques pour montrer comment la «blanchitude» et la logique anti-noire ont été normalisées. L'auteur raconte son parcours personnel et professionnel pour en apprendre davantage sur la libération des Noirs et travailler à son instauration dans les communautés noires du monde entier. Il explique comment son expérience a influencé son approche visant la libération noire dans l'enseignement des mathématiques. L'auteur indique que l'atteinte d'une libération dans l'enseignement des mathématiques est une entreprise compliquée et il présente des idées et des 
suggestions afin d'aider les adultes et étudiants noirs à mieux comprendre la situation d'un point de vue historique et actuel dans l'enseignement des mathématiques.

Larsen et Jang (2021) ont examiné les effets de l'enseignement direct et de l'apprentissage fondé sur la recherche sur la réussite des élèves du primaire en mathématiques en plus d'évaluer comment l'efficacité personnelle des élèves intervenait dans cette réussite. L'étude a aussi cherché à déterminer si le fait que des élèves aient un plan d'enseignement individualisé (PEI) affectait cette relation. Les chercheurs ont constaté une corrélation positive entre l'apprentissage fondé sur la recherche et l'efficacité personnelle alors qu'une corrélation négative a été établie entre l'enseignement direct et l'efficacité personnelle. Ces résultats indiquent que l'apprentissage fondé sur la recherche est bénéfique pour les élèves ayant des besoins particuliers et que l'on devrait encourager les enseignants à utiliser l'apprentissage fondé sur la recherche avec tous les élèves pour améliorer leurs chances de succès en mathématiques. L'étude confirme également que les enseignants devraient adopter un éventail varié de stratégies pédagogiques dans leurs salles de classe.

Chernoff (2021) poursuit sa prise de position régulière concernant l'enseignement des mathématiques. Dans ce numéro, il traite des opérations mathématiques de la messagerie Gmail sous l'angle d'un projet de recherche potentiel. Les opérations mathématiques sont une fonction de la messagerie Gmail qui visait à prévenir les courriels envoyés sous l'influence de l'alcool. L'auteur révèle ce qu'il considère comme le niveau équivalent aux compétences en maths requises pour qu'un adulte canadien en état d'ébriété puisse réussir les opérations de la messagerie. Le but des opérations mathématiques de la messagerie Gmail était d'amenuiser la possibilité que des courriels soient envoyés par des personnes en état d'ébriété. La messagerie vous envoyait des problèmes de maths à résoudre et si vous les réussissiez, vous pouviez envoyer votre courriel. Chernoff s'affaire à déterminer le niveau équivalent aux problèmes en faisant référence aux «folles minutes de calculs mathématiques». Je vous invite à vous joindre à Egan Chernoff, rédacteur en chef pour les mathématiques dans son récit pour éclaircir ce qu'il a trouvé dans un dossier laissé dans son ordinateur. Revenez nous voir dans quelques mois pour le numéro 22.1 dans lequel Egan Chernoff s'exprimera de nouveau sur d'autres sujets relatifs à l'enseignement des mathématiques.

Pyper (2021) fait le compte rendu du livre intitulé «Tensions in the borderlands: A review of Borders in mathematics pre-service teacher education (Des tensions dans les zones frontalières: un examen des frontières dans la formation initiale donnée aux enseignants en mathématiques)» (Radakovic et Limin Jao, 2020). Il indique que le livre explore les frontières dans les programmes d'études, les programmes de formation des enseignants ainsi que les interactions avec la société dans son ensemble. L'auteur utilise des systèmes d'activités pour interpréter chacun des chapitres et dans bien des cas il représente graphiquement des tensions qui existent entre les divers aspects du système d'activités. Il s'intéresse aussi aux zones frontalières, les espaces se trouvant en bordure des frontières. C'est dans ces zones frontalières que nous pouvons retrouver un potentiel de discussion et d'actions pour faire disparaître ou faire le pont avec une frontière. Pyper soutient que la mise à découvert des tensions au sein d'un système d'activités peut servir à apporter de nouveaux éléments au débat critique portant sur l'enseignement des mathématiques dans l'optique des frontières. Les auteurs du livre encouragent les lecteurs à remettre en cause les frontières dans l'enseignement des mathématiques et à considérer comment ces chapitres peuvent servir au-delà des frontières.

\section{Declarations}

Conflict of Interest L'auteur déclare qu'il n'y a pas de conflit d'intérêts. 


\section{References}

Anwandter Cuellar, N., Polotskaia, E., \& Passaro, V. (2021). La Genése de la pensée algébrique chez les enfants de trois à huit ans. Une revue de la littérature scientifique. Revue canadienne d'enseignement des sciences, des mathématiques et des technologies, 21(4).

Chernoff, E.J. (2021). Looking back at gmail's mail googles: my most maddest of mad minutes. Canadian Journal of Science, Mathematics and Technology Education, 21(4). https://doi.org/10.1007/s42330-021-00186-y

Chourney, S., \& Bakos, S. (2021). Investigating the positioning of pre-service teachers in relation to incorporating First Peoples' worldviews into mathematics teaching. Canadian Journal of Science, Mathematics and Technology Education, 21(4). https://doi.org/10.1007/s42330-021-00180-4

Davis, J. (2021). A liberatory response to antiblackness and racism in the mathematics education enterprise. Canadian Journal of Science, Mathematics and Technology Education, 21(4). https://doi.org/10.1007/s42330-021-00187-x

Husband, M. (2021). Prospective teachers working to connect their own understanding of subtraction. Canadian Journal of Science, Mathematics and Technology Education, 21(4). https://doi.org/10.1007/s42330-021-00179-x

Larsen, N.E., \& Jang, E.E. (2021). Instructional practices, students' self-efficacy and math achievement: A multi-level factor score path analysis. Canadian Journal of Science, Mathematics and Technology Education, 21(4). https://doi. org/10.1007/s42330-021-00181-3

Pirie, S., \& Kieren, T. (1994). Growth in mathematical understanding: How can we characterize it and how can we represent it? In P. Cobb (Ed.), Learning mathematics: Constructivist and interventionist theories of mathematical development (pp. 61-86). Dordrecht, NL: Kluwer.

Pyper, J. (2021). Tensions in the borderlands: a review of borders in mathematics pre-service teacher education. Canadian Journal of Science, Mathematics and Technology Education, 21(4). https://doi.org/10.1007/s42330-021-00182-2

Radakovic, N. \& Limin Jao, L. (2020). Borders in Mathematics Pre-Service Teacher Education. Springer.

Saldanha, L., \& Hatfield, N. (2021). Students conceptualizing the box plot as a tool for structuring quantitative data: a design experiment using TinkerPlots. Canadian Journal of Science, Mathematics and Technology Education, 21(4). https://doi.org/10.1007/s42330-021-00184-0

Throope Robinson, E., Lunney Borden, L., \& Carter, E. (2021). Building teacher capacity and leadership in elementary mathematics classrooms in Nova Scotia: Review of the certificate in elementary mathematics pedagogy. Canadian Journal of Science, Mathematics and Technology Education, 21(4). https://doi.org/10.1007/s42330-021-00183-1

Publisher's Note Springer Nature remains neutral with regard to jurisdictional claims in published maps and institutional affiliations. 